\title{
An Investigation of Current Dangers and Attacks Against Network Security and its Preventive Estimates Using Artificial Neural Networks.
}

\author{
Aamir Hamid Rather \\ Department of Computer Science, \\ University of Kashmir Hazratbal Srinagar -190006, \\ Email: aamir1801@gmail.com
}

\begin{abstract}
A Network can't be made sure about by utilizing security angles, for example, arranged network sniffing, network firewalls, passwords, access control, intrusion detection, interruption location, and so forth. A made sure about the system must contain all observing, early admonition gadgets, and keen avoidance strategies with intelligent techniques. This paper provides active studies of assault against Network security that has been considered and suggestions are proposed to forestall dangers and assaults dependent on ANN's (Artificial Neural Networks).
\end{abstract}

Keywords: Network Security, Attacks, ANN's, TCP, Distributed Denial of service, UDP, Threat, vulnerability, Intelligent factor.

\section{Introduction}

In network security, viable procedures ought to be executed to evade assaults. The assaults might be dynamic or inactive. The dynamic assaults incorporate adjustment of data with an intension to degenerate, decimate, or alter information in a system. The latent assault incorporates the checking of data streaming over the system. Making sure about data is a mind-boggling issue contingent on the forecast of the idea of the assault. The normal types of system assaults are collecting information, information adjustment, identification phishing attacks, attacks based on passwords, denial of service (Dos), middle attackman, breached key attacks, trojan assaults, application layer level assaults, etc. [8] Even more testing assault is the distributed denial of service (DDoS). The Denial of Service (Dos) attack is an attack strategy through which an individual can render the framework obsolete or, in essence, impede this same framework for genuine customers by overburdening the assets and nobody can get there. One can isolate the Dos assaults into three different clusters [12].
1.1 Bandwidth Attacks: Bandwidth assaults are utilized to expend assets, for example, transmission capacity or hardware throughput. High information volume assaults can devour all accessible data transmission between an ISP and its site. The connection tops off, and genuine traffic eases back down. Breaks may happen, causing retransmission, producing significantly more traffic. An essential flood assault may utilize UDP or ICMP parcels to just expend all accessible transmission capacity. I.e., an assault could comprise of TCP or crude IP bundles as long as the traffic is steered to claim to organize. A simple assault on the use of data transfer resources will abuse the throughput ranges of servers or system gear by relying on high packet rates[11], sending out a huge number of small plots.

1.2. Protocol Attacks: - It utilizes the normal conduct of conventions, TCP, UDP, and ICMP for a bit of leeway for the aggressor, for example. Models are SYN flood attacks in which the attacker floods the casualty with bundles of TCP SYN, and the casualty designates assets to identify obvious approaching modifications. Smurf is a lopsided reflector assault that objectives a powerless system 
communicate address with ICMP ECHO REQUEST packets.

2. Software Vulnerability Attacks: - There are legal attacks that exploit flaws in network infrastructure, such as a database server or a fundamental TCP / IP block. There are a few versions: -

$>$ Teardrop misuses TCP/IP stacks that don't appropriately deal with covering IP pieces.

$>$ LAN designs IP packets which have configured the source node and port to be identical to the target address and port.

$>$ Ping of Death (POG) delivers a large, lone ICMP reverberation demand package to the goal.

Denial of service (DoS) assaults might present itself as a viable as a result of a blending of impacts. For example, an attack that does not completely consume transmission capacity or transmission overload throughput may be feasible because it creates sufficiently deformed congestion to malfunction a specific service, such as a web application or server software.

DDoS attacks involve hacking through a large number of computers all over the Internet.[5] An attacker transmits DDoS assaults with a few devices. And thereafter, the instigator enters into many computers to execute an assault on an objective device or computer networks simultaneously. DDoS assault makes it hard to recognize because assaults start from several IP addresses. At that point the aggressor introduces Distributed denial of service attacks programming on them, allowing them to take control of all of these corrupted devices to dispatch composite assaults to the premises of the victims. Such attacks typically consume data transmission power, move processor maximum, or device stack properties, and interrupt the functionality of the network to the individual concerned.

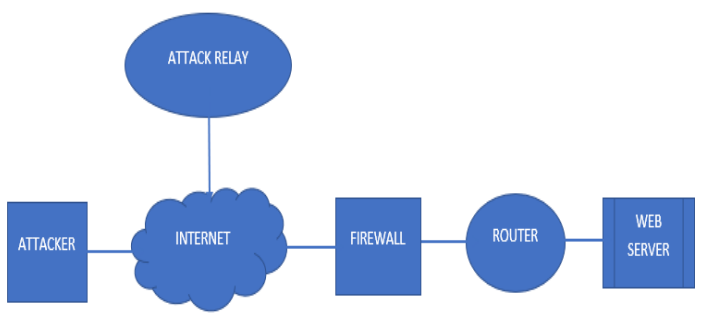

Fig1- Network Diagram -DDoS Attack
DDoS is a combination of DoS attacks to punish the target host by advancing its performance from different hosts [8]. The term is used when the origins of an attack come from several sources, rather than from one origin. Such invasions can not be eradicated by filtering the source IP, because they start with the numerous handlers from many points coordinated. Big equipment includes Mstream, Trinoo, Stacheldraht, Pipe, Tribe Flood Network (TFN). The key methods used to execute a denial-of-service attack (DoS) assaults are:-

1. Ping Flood: - In this type of attack, the assailant sends an IP packet bigger than 65,535 bytes authorized by the internet protocol. In this scenario, TCP / IP is suitable for atomization. This allows you to split a single IP packet into smaller sections. The process involves forwarding a large ICMP packet to the preferred destination by an inimical machine, the destination machine collects the ping into pieces and initiates building the packet. Since the size constraint of the packet, as it reappears, it becomes impossible for the buffer to process and hence overflows. Most Operating systems don't have the programmed information regarding dealing with big packets. Eventually, they freeze, crash, and the system restarts. This type of approach is particularly damaging because the identification of the attacker who forwards big ICMP packets is easily diminished. After all, the assailant has only the requirement of IP address to execute his attacks. [6]

2. SYN - Flood attack: -Uses common programming to load the system with demands for the half-open link. It is spread to a limited utility, the $\mathrm{C}$ source, to which, when used against the Unix server, will suspend the server's work temporarily.

3. Land Exploit: - A technique to send a spoofed packet in association with SYN flag which is then used to provide the handshake among the client and the host and sets it to a specific port which is active and an available to listen. On the contrary, If the specific packet is organized for the same destination and the IP address of the source sent to the machine, it will fool the transmission machine into thinking that it is sending a message to self by IP spoofing, which crashes the machine.

The main hacking tools for DDoS attacks are: - 
1. Trinoo: -Trinoo is a set of computer programs to conduct a DDoS attack which runs on below Transmission Control Protocol (TCP) ports.

- A Master Attacker: 27665 / TCP

- Daemon Master: 27444 / UDP

- Master daemon:31335 / UDP

It is compromised by buffer overrun bug in RPC services on Solaris 2.x system: Stadt, Cmsd, ttd observed. The Trinoo daemon was based on UDP, shells with remote commands were passwordprotected and operated on broken frameworks.

2. TFN: - Tribe Flood Network uses an ace program to interact with the assault located across various systems. TFN dispatches enabled DoS assaults that are especially challenging to contend with because that can generate various forms of assaults and can yield packets of IP addresses of fake origins. A portion of the assaults that TFN will lead involves the flooding of UDP Flood, TCP SYN Flood, ICMP ecorequest, and ICMP direct communication.

3. Stacheldraht v2.667: It consolidates the highlights of TFN and Trinoo yet includes encryption between daemons. Stacheldraht utilizes TCP and ICMP of the accompanying ports.

- Customer to Handler: TCP 16660

- Handler by and to Agents: ICMP 65000

- The DDoS assaults are not the same as sniffer assaults that grab network logins and passwords that go around.[13]

DoS assaults may be identified through its Ramp-up behaviour. It is an adjustment of the assault's volume in the rush hour gridlock as a function of time. In a multisource attack, a master promptly or at some later time periodically enacts a colossal number of Zombies. At the point when seen near the person in question, Zombie's confiscated enactment increases the force of the attack. Similarly, the spectral qualities of assaults can be read for characterizing binary and multi-source attacks.

The spectral intensity can indeed be discovered for fixed fragments by playing the discrete-time game. Fourier transforms the attack streams on the autocorrelation function.[4] The $\mathrm{r}(\mathrm{k})$ autocorrelation group at $\log \mathrm{k}, \mathrm{t}=0$

$$
\mathrm{C}(\mathrm{k})=1 / \mathrm{N}-\mathrm{K} \sum\left[\left(\mathrm{x}(\mathrm{t})-\mathrm{x}^{-}\right)\left(\mathrm{x}(\mathrm{t}+\mathrm{k})-\mathrm{x}^{-}\right)\right]
$$

where $x^{-}$is the mean of $x(t)$ and $N$ is the length of the assault stream $x(t)$. The force range $s(f)$ of the assault got by the discrete-time Fourier change of the autocorrelation succession of length $\mathrm{m}$.

$$
\begin{aligned}
& \mathrm{m} \\
& \mathrm{s}(\mathrm{f})=\sum \mathrm{r}(\mathrm{k}) \mathrm{e} \\
& \mathrm{N}-\mathrm{k} 2 \pi \mathrm{fk} \mathrm{t}=0
\end{aligned}
$$

The highest recurrence discernible through this strategy is 500 hertz for one millisecond of the moment and even the Fourier change is well proportioned.

IDS based on deployment medium:

To distinguish interference, various methodologies include Data processing, Clustering, Naive Bayesian Classifiers (NBC), Bayesian Networks, Hidden Markov systems, Trees Decision, ANNs, supporting vector machines, etc.[11].

Enhanced IDS may very well have accompanying useful eligibility criteria.,

$>$ It ought to constantly screen and address intrusion.[7]

$>$ It might likewise be intended to redress or fix essential interruption.

$>$ It should discover and kill composed attacks.[6]

$>$ It should be able to take extra traffic loads in its capacity.[9]

$>$ It ought to forestall harm to the system and its devices.[10]

$>$ It regulates, evaluates and helps to prevent a favourable objective for hackers.

An ANN specialist is intended to beat the accompanying confinements: -

$>$ Exorbitant information traffic [11].

$>$ Intruders can perform inclusion and avoidance assaults. Ordinarily, information assortment is acted in a host unique about the one where analyses are performed.

The new idea ought to have the accompanying qualities
$>$ Insight
$>$ Adaptability [9]
$>$ Portability
$>$ Quick response
$>$ Early admonition framework 
ISSN(Online) : 2456-8910

International Journal of Innovative Research in Applied Sciences and Engineering (IJIRASE)

Volume 4, Issue 1, DOI: 10.29027/IJIRASE.v4.i1.2020. 598-602, July 2020

$>$ Update

$>$ Invulnerability to assaults.

The new structure of IDS ought to have some unique capacities, for example, Event material, sifting capacity, Identification work, checking validity work with ANN Networks of knowledge operators, portability specialists, adaptability specialist and so on.

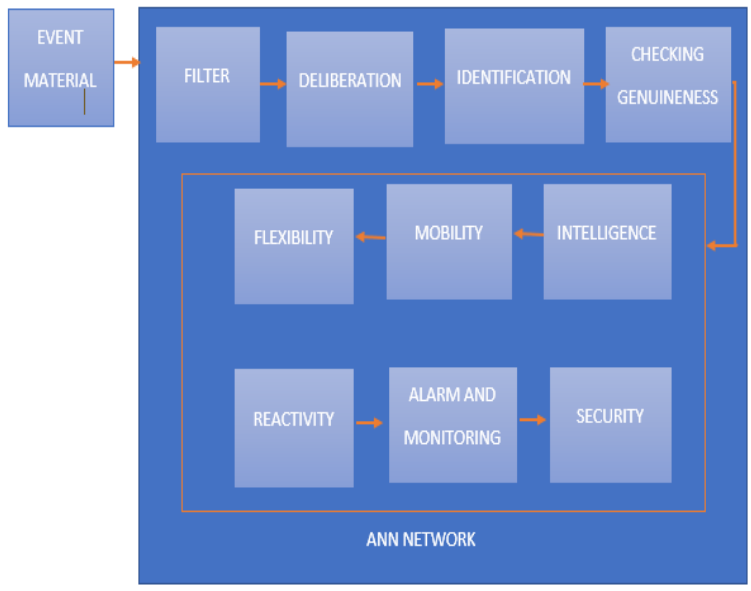

\section{Fig2. The new design of IDS}

The separating events can be put away in the consideration function. It associates with the distinguishing proof operator where it checks the filtered occasion with security strategies, consents, refusals, and so forth. The validity will be checked after distinguishing proof. Further utilizing the ANN Network framework, a clever operator will additionally distinguish and explain the occasion with the assistance of portability, adaptability capacities. At long last, the security operator might be in a situation to make sure about any sort of danger.

The implemented smart artificial neural networkbased IDS:

The IDS might be intended to choose at a suitable period to maintain a strategic distance from botches and along with these lines' successful location and anticipation of the objective framework. The proposed block outline is as under:

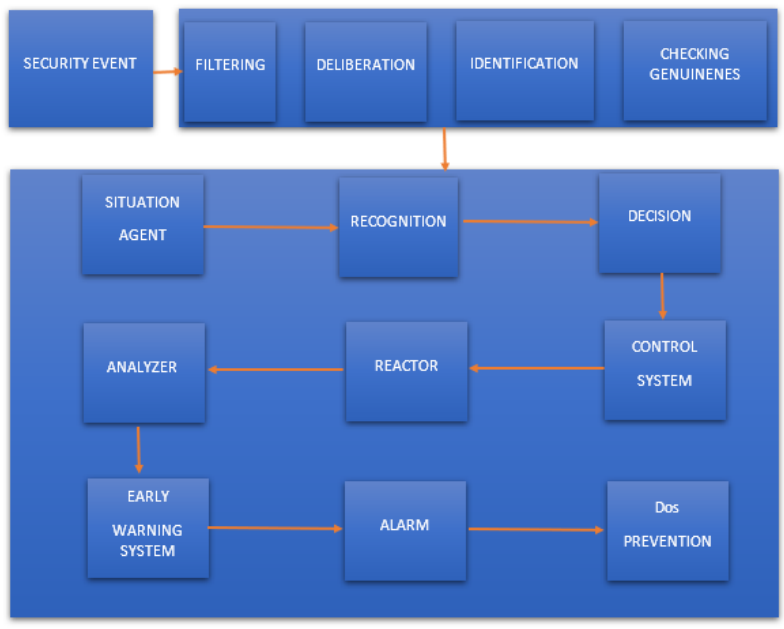

Fig3. Network security using ANN

The approaching occasions are sifted and gone to the ANN to conclude as per the current situation. The ANN may utilize information, Recognition, Situation, lastly choose to relieve any type of Denial of Service assaults.

\section{Conclusion}

Concluding this research paper, I referenced various essential Denial of Service assaults greater focus on early scientific work on the point of the problem. The suggested security of the board design dependent on the idea of Intelligent specialists in the intersection with Artificial Neural Networks takes a choice on the security aspect of Denial of Service assaults. The security measurements on the proposed arrange outline get to access control, confirmation, nonrevocation, information classification, correspondence security, information integrity, accessibility, availability, privacy, and protection.

\section{References}

[1Ramasubramanian, P., and Arputharaj Kannan. "Intelligent Multi-Agent Based Multivariate Statistical Framework for Database Intrusion Prevention System." Int. Arab J. Inf. Technol. 2, no. 3 (2005): 239247.

[2] Dewan, Prashant, Partha Dasgupta, and Vijay Karamcheti. "Defending against Denial of Service attacks using Secure Name Resolution." In Security and Management, pp. 675-681. 2003.

[4]" Jain, Payal, Juhi Jain, and Zatin Gupta. "Mitigation of denial of service (DoS) attack." IJCEM International Journal of Computational Engineering \& Management 11 (2011): 2230-7893. 
[5Manninen, Matti. "Using artificial intelligence in intrusion detection systems." Helsinki University of Technology 13 (2002).

[6] Aamir, Muhammad, and Muhammad Arif. "Study and performance evaluation on recent DDoS trends of attack \& defense." International Journal of Information Technology and Computer Science 5, no. 8 (2013): 5465.

[7] Kukielka, Przemyslaw, and Zbigniew Kotulski. "Adaptation of the neural network-based IDS to new attacks detection." arXiv preprint arXiv:1009.2406 (2010).

[8] Noaman, Khaled MG, and Hamid Abdullah Jalab. "Data security based on neural networks." Task Quarterly 9, no. 4 (2005): 409-414.

[9] Gandhi, Meera, and S. K. Srivatsa. "Detecting and preventing attacks using network intrusion detection systems." International Journal of Computer Science and Security 2, no. 1 (2008): 49-58.

[10] Network and computer security tutorial version 0.4.0 "https://www.tutorialspoint.com/network security/index.htm" issue 2019.

[11] Cisco certified network professional guide Vol 1 Issue 2019 and Page No 147.

[12] Idris, Norbik Bashah, and Bharanidlran Shanmugam. "Artificial intelligence techniques applied to intrusion detection." In 2005 Annual IEEE India ConferenceIndicon, pp. 52-55. IEEE, 2005. 